\title{
Protective effects of quercetin on UVB irradiation-induced cytotoxicity through ROS clearance in keratinocyte cells
}

\author{
XIANBING ZHU ${ }^{1,2^{*}}$, NING $\mathrm{LI}^{2 *}$, YILING WANG ${ }^{2}, \mathrm{LI} \mathrm{DING}^{2}, \mathrm{HOUJIE} \mathrm{CHEN}^{2}, \mathrm{YEHUI} \mathrm{YU}^{2}$ and XIAOJUN SHI ${ }^{1,2}$ \\ ${ }^{1}$ School of Life Sciences, Tsinghua University, Beijing 100084; ${ }^{2}$ The Shenzhen Key Laboratory of Gene and \\ Antibody Therapy, Graduate School at Shenzhen, Tsinghua University, Shenzhen, Guangdong 518055, P.R. China
}

Received August 19, 2016; Accepted October 13, 2016

DOI: 10.3892/or.2016.5217

\begin{abstract}
Human skin is the body's largest organ that protects against diverse environmental injuries. However, ultraviolet (UV) radiation, which induces a transient increase in the intracellular level of reactive oxygen species (ROS) and leads to a variety of injuries and various skin diseases, has deleterious effects on living organisms. Quercetin is a naturally occurring compound with strong antioxidant action and can successfully scavenge free radicals. In the present study, we investigated the effects and the mechanism of quercetin on UVB-induced cytotoxicity in keratinocyte (HaCaT) cells. The results of this study showed that quercetin $(20 \mu \mathrm{M})$ significantly blocked UVB irradiation $\left(15 \mathrm{~mJ} / \mathrm{cm}^{2}\right)$-induced intracellular ROS generation. In addition, the ROS clearing ability of quercetin prevented cell membrane and mitochondria from ROS attack and inhibited cell membrane fluidity decrease and mitochondrial membrane depolarization. Moreover, the outflow of cytochrome $c$ and apoptosis were markedly inhibited. These results suggest that the protective effect of quercetin against UVB irradiation-induced toxicity is mainly mediated by the ROS scavenging ability. Thus, quercetin is a potential agent against UVB irradiation-induced skin damage.
\end{abstract}

\section{Introduction}

The human skin is the most exposed organ of the human body and protects against diverse environmental injuries. Meanwhile, human skin is also at risk of continuous and repetitive environmental damage. Solar ultraviolet (UVA, UVB and UVC) radiation is a sort of high-energy electromagnetic radiation that is thought to have xenotoxic effects on all living organisms. Particularly UVB $(280-320 \mathrm{~nm})$, which is

Correspondence to: Dr Xiaojun Shi, School of Life Sciences, Tsinghua University, Beijing 100084, P.R. China

E-mail: shixj@sz.tsinghua.edu.cn

\section{"Contributed equally}

Key words: lipid peroxidation, mitochondrial, cytochrome $c$, apoptosis, cell membrane fluidity the main component of solar $\mathrm{UV}$, is incapable of penetrating deeply into the skin and it only affects the epidermis, the superficial layer of the skin, which is composed predominantly of keratinocytes (1). UVB irradiation of the skin can result in a variety of injuries and leads to various skin diseases (2). It is reported that UVB irradiation has been shown to induce a transient increase in the intracellular level of reactive oxygen species (ROS) in human skin, particularly in keratinocytes (HaCaT) (3-9). These reactive oxygen species (ROS), including hydroxyl radicals, superoxide radicals, peroxyl radical and their active precursors namely singlet oxygen, hydrogen peroxide and ozone (10), attack the cell membrane and cellular organelles resulting in lipid peroxidation and organelle damage. UVB irradiation of $\mathrm{HaCaT}$ cells results in a post-irradiation-dependent increase in ROS and subsequent cell membrane fluidity decrease and mitochondrial membrane depolarization (1). Plasma membrane fluidity affects a number of cellular functions, such as carrier-mediated transport, the properties of certain membrane-bound enzymes and cell growth. Membrane fluidity decrease has deleterious effects on cells and triggers apoptosis (11). Mitochondria are both the major producers and targets of ROS in mammalian cells (12). UVB-induced ROS can attack mitochondria and result in mitochondrial structure change and the collapse of mitochondrial membrane potential $(\Delta \Psi \mathrm{m})$ of mitochondria and the subsequent outflow of apoptosis-inducing factors and apoptosis. UVB irradiation-induced ROS play an important role in the occurrence of apoptosis (10) and solar radiation is a crucial factor that deeply affects this specialized epithelium (13) and skin diseases.

Thus, reducing intracellular ROS levels may represent an effective strategy for preventing UVB-induced $\mathrm{HaCaT}$ apoptosis and skin damage. In recent years, naturally occurring compounds, flavonoids, which are abundant in fruits, vegetables, green tea, and red wine and possess a variety of biological activities including antioxidant, have gained considerable attention as protective agents against UVB-induced skin damage via scavenging ROS. Quercetin is one of the flavonoids derived from numerous fruits and vegetables with high antioxidant action and provides protection against UV-induced damage to plants. Studies have reported that quercetin has diverse pharmacological activities, and the protective effects of quercetin have been attributed to the inhibition of key signaling enzymes involved in the regulation of 
cell proliferation, angiogenesis and apoptosis (14). In addition, quercetin has strong antioxidant activity and can scavenge free radicals and inhibit lipid peroxidation and metal ion chelation (15-22). Due to the antioxidant properties of quercetin, we hypothesized that quercetin may provide protection against ROS attack on the cell membrane and mitochondria and block lipid peroxidation and release of apoptosis-inducing factors from mitochondria via ROS clearing activity.

In the present study, we investigated the protective effect of quercetin against ROS-induced cell membrane and mitochondrial damage in UVB-irradiated $\mathrm{HaCaT}$ cells. The results showed that quercetin effectively cleared UVB irradiation-induced ROS in the HaCaT cells and blocked ROS-induced lipid peroxidation, mitochondrial membrane depolarization and subsequent outflow of apoptosis-inducing factors and apoptosis.

\section{Materials and methods}

Materials. Quercetin was purchased from Sigma Aldrich (St. Louis, MO, USA). 4', 6-Diamidino-2-phenylindole dihydrochloride (DAPI) was purchased from Sangon Biotech (Shanghai, China). DCFH-DA, Dio, JC-1 were purchased from Beyotime Biotechnology (Shanghai, China). All other chemicals and reagents of the highest quality were commercially available and used as received. Antibodies against cytochrome $c, \mathrm{Bcl}-2$, Bax, caspase-3 and PARP were purchased from Cell Signaling Technology Inc. (Beverly, MA, USA).

Cell culture and UVB irradiation. Human skin keratinocytes, HaCaT, were cultured in Dulbecco's modified Eagle's medium (DMEM; Gibco BRL, Grand Island, NY, USA) supplemented with $10 \%$ heat-inactivated fetal bovine serum (FBS; Biological Industries Ltd., Israel) at $37^{\circ} \mathrm{C}$ in $5 \% \mathrm{CO}_{2}$. The cells were incubated with or without quercetin for $8 \mathrm{~h}$ prior to UVB irradiation. Then, the cells were washed with phosphate buffered saline (PBS; pH 7.4) and irradiated using a microprocessor-controlled UV Crosslinker (XL-1000; Spectrolinker ${ }^{\mathrm{TM}}$, Westbury, NY, USA). The irradiation intensity was monitored by a UVB radiometer (Beijing Normal University, China). Immediately after UVB irradiation, the cells were returned to the incubator and incubated with drug-free media for the indicated times.

Assessment of cell viability. The cell viability was determined using MTT assay. The cells were seeded in 96-well plates at a concentration of 5,000 cells $/ \mathrm{ml}$. The cells underwent pretreatments with quercetin mixed with dimethyl sulfoxide (DMSO) at various concentrations. Before irradiation, the cells were incubated for $18 \mathrm{~h}$ and then, after irradiation, the cells were incubated for the indicated times. Following this, $20 \mu \mathrm{l}$ MTT $(2 \mathrm{mg} / \mathrm{ml}$ ) was added, and the cells were incubated for another 4 h. Finally, $200 \mu \mathrm{l}$ DMSO was added to dissolve the crystals, and the plates were read immediately on a plate reader at a test wavelength of $490 \mathrm{~nm}$.

Measurement of intracellular ROS levels. Cells grown on coverslips in 6-well plates were exposed to the indicated treatments. Quercetin was applied, and the incubation time after irradiation was $24 \mathrm{~h}$. Cells were incubated with DCFH-DA according to the instructions and immediately examined by confocal laser scanning microscopy (Olympus FV1000-IX81, Tokyo, Japan) using an argon laser with a 488-nm excitation band. The laser intensity, pinhole diameter and photomultiplier settings were kept constant for every experiment. For quantitative analysis, the ROS levels were examined using a Partec PAS III flow cytometer (Partec, Munster, Germany).

Fluorescence recovery after photobleaching (FRAP). FRAP analysis was performed as previously described (23-25). Briefly, after incubation with quercetin for $8 \mathrm{~h}$, the cells were irradiated with UVB $\left(15 \mathrm{~mJ} / \mathrm{cm}^{2}\right)$. Twelve hours after irradiation, the cells were incubated with the Dio probe according to the protocol, and photobleaching was immediately conducted by confocal laser scanning microscopy (FV1000-IX81; Olympus, Tokyo, Japan) (with excitation band $488 \mathrm{~nm}$, absorption band $530 \mathrm{~nm}$, bleaching band $405 \mathrm{~nm}$, power $91 \%$, time $1 \mathrm{sec})$.

$$
\begin{gathered}
\mathrm{R}(\text { recovery })=(\mathrm{F} 1-\mathrm{F} 0) /(\mathrm{F} 2-\mathrm{F} 0) \times 100 \% \\
\mathrm{D}(\text { diffusion coefficients })=\beta \omega^{2} / 4 \mathrm{t}_{1 / 2}
\end{gathered}
$$

where F2 is the fluorescence intensity after the recovery, F1 is the the fluorescence intensity before the photobleaching, F0 is the fluorescence intensity immediately after the photobleaching, $\beta$ is the conversion factor, $\omega$ is the radius of the bleached area and $t_{1 / 2}$ is the half time $\left(t_{1 / 2}\right)$ of recovery into the bleached region.

Measurement of intracellular malondialdehyde (MDA) level. Cells were seeded in 6-well plates at a concentration of $1 \times 10^{7}$ cells $/ \mathrm{ml}$. After the cells had undergone the indicated treatments, quercetin was applied, and the incubation time after irradiation was $24 \mathrm{~h}$. The MDA levels were estimated using the commercially available colorimetric MDA-586 assay kit from Beyotime Biotechnology. The absorbance was determined using a microplate reader (ELX808; Bio-Tek Instruments, Inc., Winooski, VT, USA) at a wavelength of $586 \mathrm{~nm}$. The protein content was measured using a BCA assay kit from Beyotime Biotechnology.

Detection of mitochondrial membrane depolarization. Cells were seeded into 6-well plates until reaching $80 \%$ confluency. Quercetin was applied, and the incubation time after irradiation was $24 \mathrm{~h}$. Cells were stained with JC-1 according to the manufacturer's protocol and were immediately examined by confocal laser scanning microscopy (FV1000-IX81; Olympus). The laser intensity, pinhole diameter and photomultiplier settings were kept constant for every experiment. For quantitative analysis, the $\Delta \Psi \mathrm{m}$ was examined using a Partec PAS III flow cytometer (Partec).

Immunocytochemical staining. Cells were seeded in a 12-well plate with a cover glass and incubated at $37^{\circ} \mathrm{C}$ with $5 \% \mathrm{CO}_{2}$ overnight. Quercetin was added to the medium and incubated for $8 \mathrm{~h}$. Then the cells were washed and irradiated with the indicated irradiation intensity. Twenty-four hours after irradiation, the cells were fixed, permeabilized and the immunofluorescence staining with anti-Tom antibody was performed.

Immunoblotting. Immunoblot analysis was performed as previously described (26). Briefly, the cell lysates were resolved 
A<smiles>O=c1c(O)c(-c2ccc(O)c(O)c2)oc2cc(O)cc(O)c12</smiles>

B

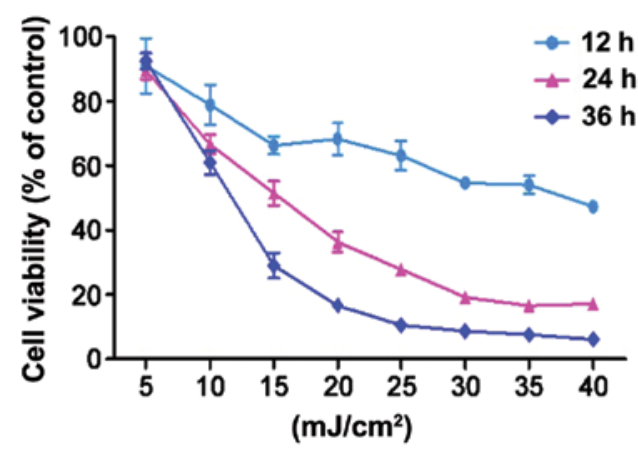

C

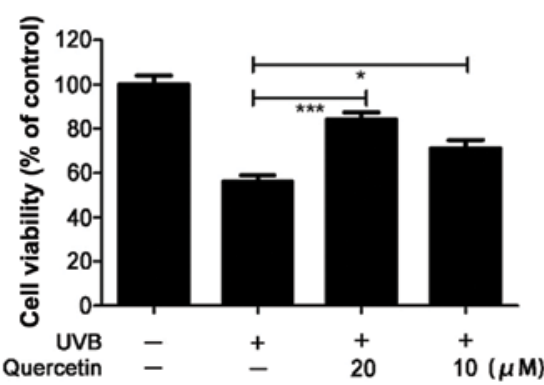

Figure 1. Protective effect of quercetin on cell viability following UVB irradiation. (A) Chemical structure of quercetin (3,3',4',5,7-pentahydroxylflavone). (B) After treatment with increasing doses of UVB irradiation for 12,24 and $48 \mathrm{~h}$, the effect of UVB on cell viability was determined using the MTT assay. (C) After preincubation with quercetin at the concentrations of 20 or $10 \mu \mathrm{M}$ for $8 \mathrm{~h}$, the cells were irradiated with UVB at the dose of $15 \mathrm{~mJ} / \mathrm{cm}^{2}$. Cell viability was determined $24 \mathrm{~h}$ post-irradiation. The data represent the means $\pm \mathrm{SD}$ of three independent experiments. ${ }^{*} \mathrm{P}<0.05$ and ${ }^{* * *} \mathrm{P}<0.001$.

on $12 \%$ SDS-PAGE and analyzed by immunoblotting using cytochrome $c$, Bcl-2, Bax, caspase-3 and PARP antibodies, followed by enhanced chemiluminescence (ECL) detection (Thermo Scientific).

Statistical methodology. The data are presented as mean \pm SE. Significant differences between two groups were analyzed with the Student's t-test. One-way analysis of variance (ANOVA) was applied to analyze differences in data of biochemical parameters among the experimental groups, followed by Dunnett's test for pair-wise multiple comparisons. $\mathrm{P}<0.05$ was considered to indicate a statistically significant difference.

\section{Results and Discussion}

Quercetin increases the viability of UVB-treated HaCaT cells. Quercetin is known to possess antioxidant properties due to the o-hydroxy structure in the $\mathrm{B}$ ring, the 2-3 double bond in conjugation with the 4-oxo function in the C-ring and the 3- and 5-OH groups with the 4-oxo function in the $\mathrm{A}$ and $\mathrm{C}$ rings (Fig. 1A) (27). It is reported that quercetin reduces cell death in cutaneous tissue-associated cell types (28). Thus, we investigated the protective effect of quercetin against UVB irradiation-induced damage in $\mathrm{HaCaT}$ cells. The cell viability (\% of the control) in the UVB-irradiated HaCaT cells was evaluated by MTT assay. UVB irradiation caused cell death in a time and dose-dependent manner (Fig. 1B). Pretreatment with quercetin significantly increased cell viability $24 \mathrm{~h}$ post-irradiation at the dose of $15 \mathrm{~mJ} / \mathrm{cm}^{2}$ (Fig. 1C). This demonstrated that quercetin could rescue $\mathrm{HaCaT}$ cells from UVB irradiation-induced cell injury. As UVB radiation is known to have deleterious effects on cells via ROS $(29,30)$, we hypothesized that one of the possible mechanisms for the protective effect of quercetin could be attributed to ROS scavenging capacity.

Quercetin reduces UVB irradiation-induced intracellular ROS levels. To investigate whether quercetin reduces UVB-induced ROS generation, a fluorescence assay using DCFH-DA was performed. DCFH-DA is hydrolyzed by intracellular esterase and is converted to nonfluorescent DCFH, which is oxidized to highly fluorescent DCF in the presence of ROS $(10,31)$. The fluorescence intensity is a measurement of intracellular ROS activity. The qualitative assessment of the cellular level of ROS was conducted by confocal laser scanning microscopy. UVB irradiation of $15 \mathrm{~mJ} / \mathrm{cm}^{2}$ significantly increased green fluorescence intensity (Fig. 2A). When the cells were pretreated with quercetin, the DCF fluorescence intensity was clearly reduced and the group treated with $20 \mu \mathrm{M}$ quercetin had a weaker fluorescence than the $10 \mu \mathrm{M}$ quercetin-treated group. Fig. 2B shows the quantitative analysis of the cellular ROS levels and the bar graph in Fig. 2C displays the fluorescence intensity of the flow cytometric results. The results demonstrated that UVB irradiation caused intracellular ROS generation and quercetin effectively reduced the intracellular ROS generated by UVB irradiation. Furthermore, N-acetyl-cysteine (NAC), an effective ROS clearing agent, effectively scavenged UVB-induced ROS in the HaCaT cells (Fig. 3A and B).

Quercetin prevents cells from ROS-induced membrane fluidity decrease and lipid peroxidation. UVB irradiation-induced ROS may attack the cell membrane and result in lipid peroxidation and cell membrane fluidity decrease. As quercetin effectively reduced UVB irradiation-induced ROS generation, we hypothesized that quercetin prevents cells from ROS-induced membrane fluidity decrease and lipid peroxidation. FRAP is a technique that monitors the diffusion of fluorescent molecules into a region in which fluorophores have been irreversibly photobleached by a high intensity laser pulse. A fluorescent probe (Dio) was used to stain the cell membrane, and the membrane fluidity was detected via lateral diffusion by monitoring the recovery of fluorescence into a region following a single photobleaching event. The fluorescence intensity before the photobleaching, the fluorescence intensity immediately after photobleaching and the fluorescence intensity after the recovery, respectively 
A
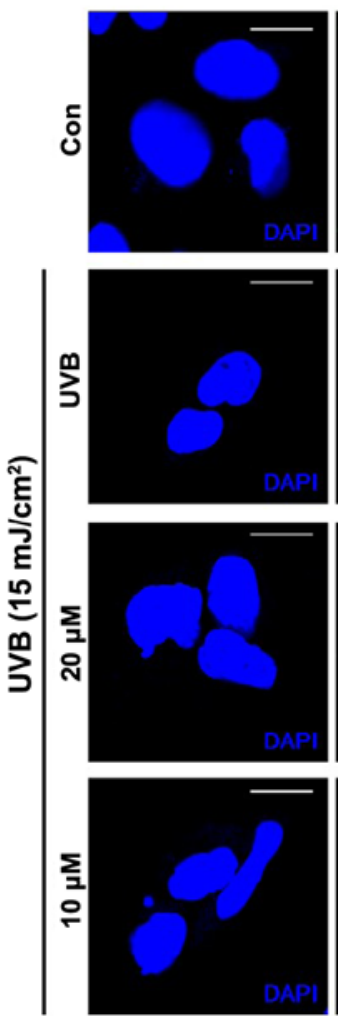
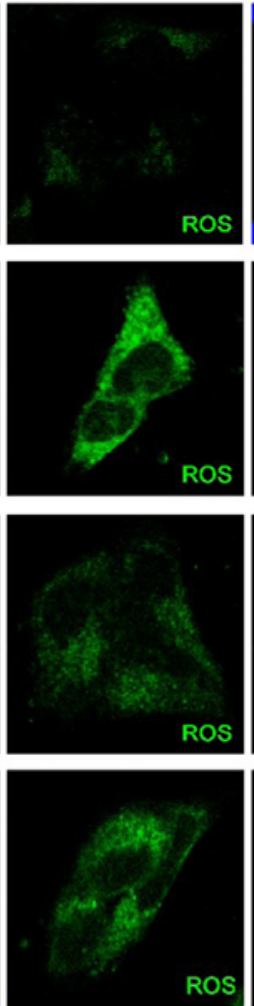
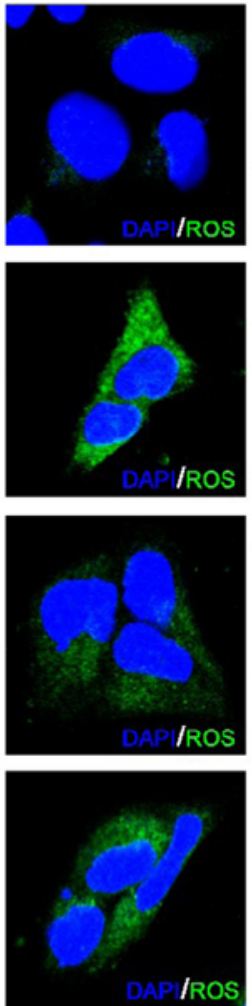

B a
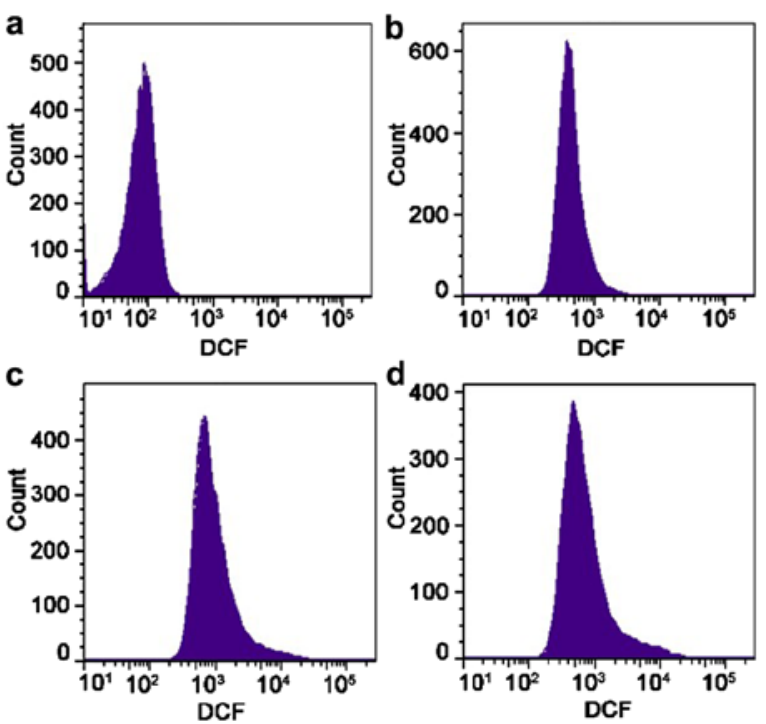

C

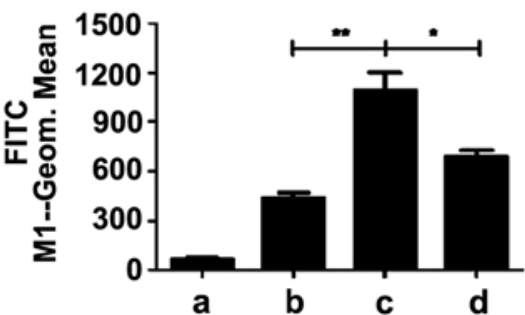

Figure 2. Effect of quercetin on intracellular ROS clearing in cultured HaCaT cells. HaCaT cells were pretreated with 10 or $20 \mu \mathrm{M}$ quercetin for $8 \mathrm{~h}$ Twenty-four hours post-irradiation, the intracellular ROS levels were determined using the fluorogenic probe DCFH-DA. (A) The intracellular ROS levels were qualitatively determined via confocal laser scanning microscopy. Scale bars, $10 \mu \mathrm{m}$. (B) The intracellular ROS levels were quantitatively determined via flow cytometry. (a) No probe loaded; (b) probe loaded and no UVB treatment; (c) probe loaded and UVB treatment; (d) probe loaded, UVB treatment, and quercetin pretreatment. (C) The relative intensity of DCF fluorescence is shown in the bar graph. The data represent the means \pm SD of three independent experiments. ${ }^{*} \mathrm{P}<0.05,{ }^{* *} \mathrm{P}<0.01$.

A a

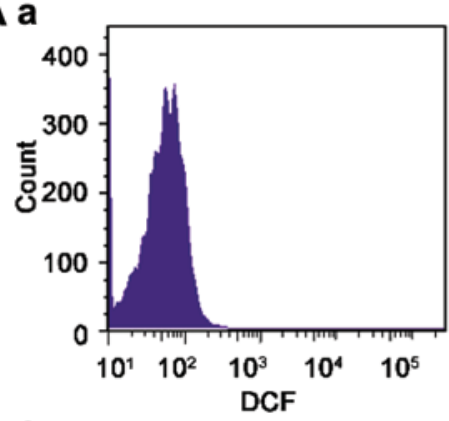

C

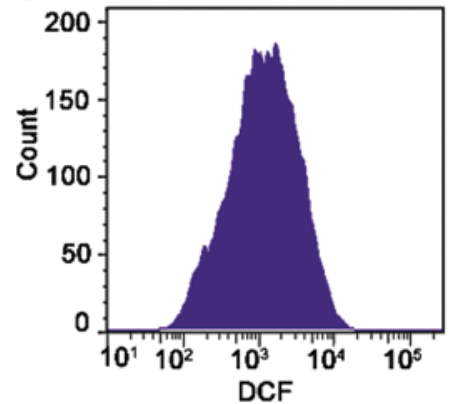

b

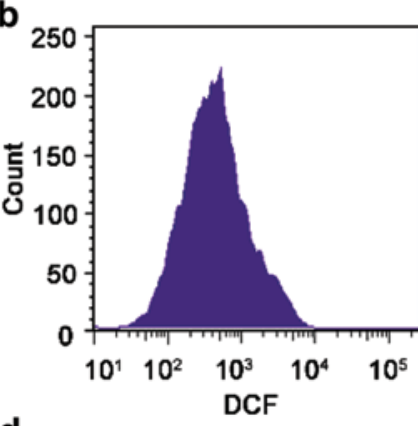

d

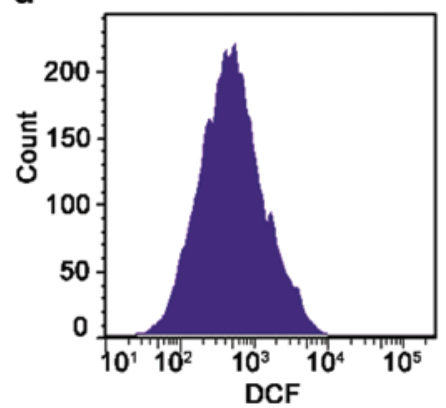

B

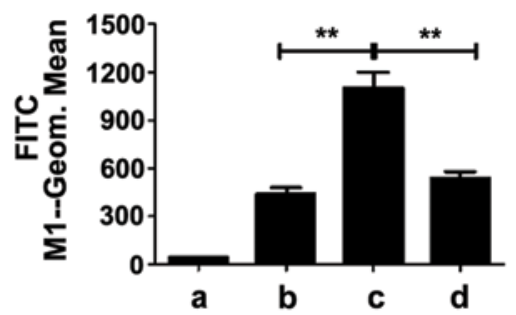

C

$\begin{array}{llll}\text { NAC } & - & - & + \\ \text { UVB } & - & + & +\end{array}$

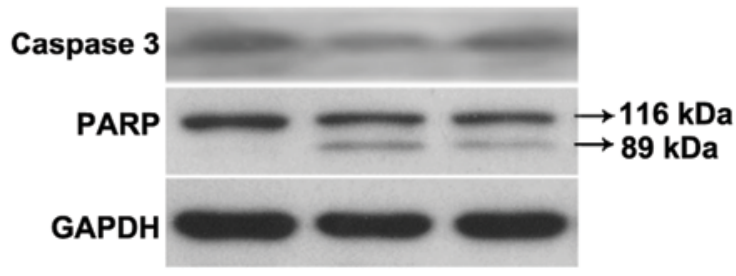

Figure 3. N-acetyl-cysteine (NAC) effectively scavenges UVB-induced ROS and inhibits cell death. HaCaT cells were pretreated with 5 mM NAC for $8 \mathrm{~h}$. Twenty-four hours post-irradiation, the intracellular ROS levels were determined using the fluorogenic probe DCFH-DA. (A) The intracellular ROS levels were quantitatively determined via flow cytometry. (a) No probe loaded; (b) probe loaded and no UVB treatment; (c) probe loaded and UVB treatment; (d) probe loaded, UVB treated and NAC pretreatment. (B) The relative intensity of DCF fluorescence is shown in the bar graph. (C) The protein expression of caspase- 3 and PARP. The data represent the means \pm SD of three independent experiments. ${ }^{* *} \mathrm{P}<0.01$. 

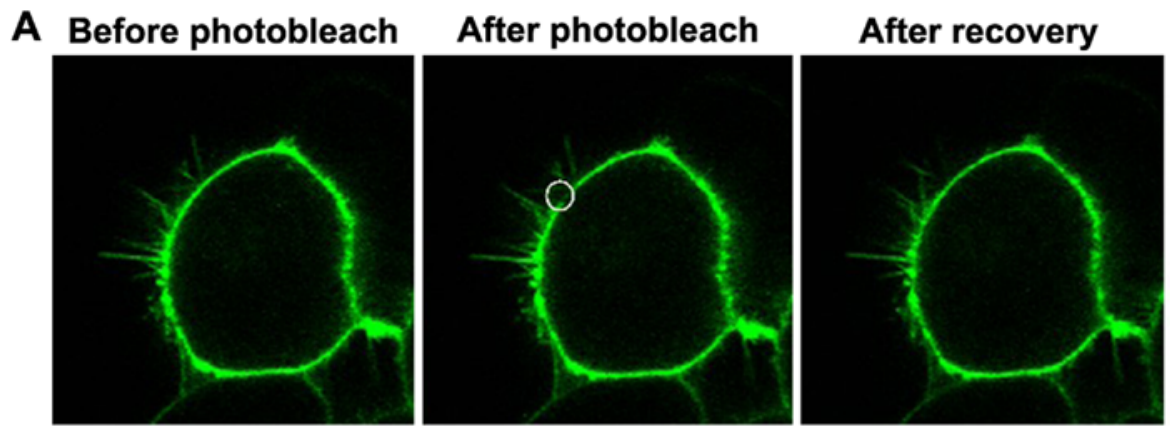

Ba

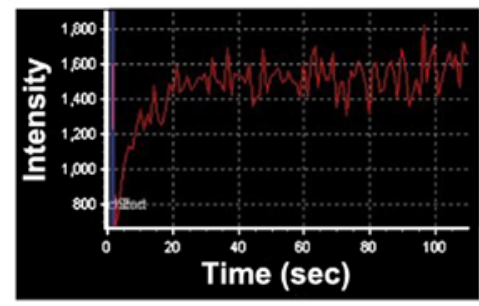

b

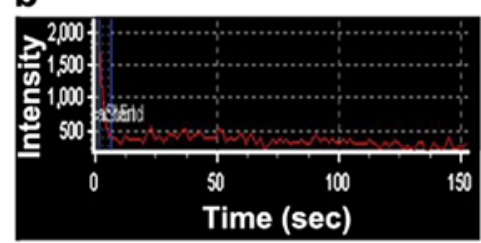

C

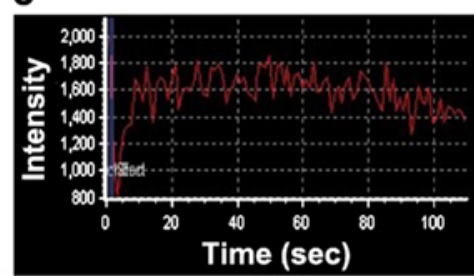

d

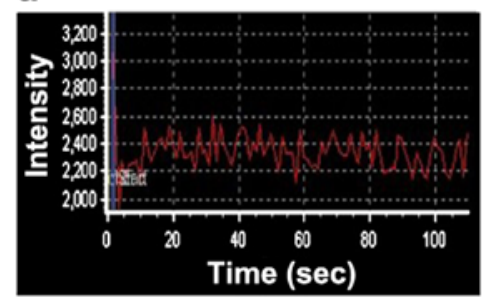

C

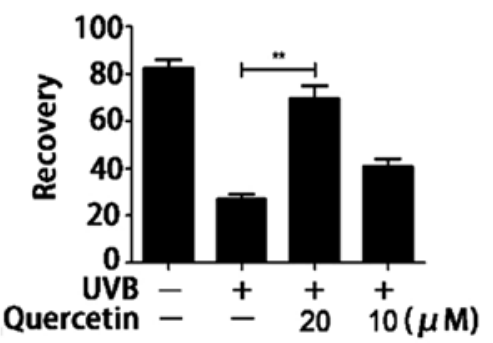

D

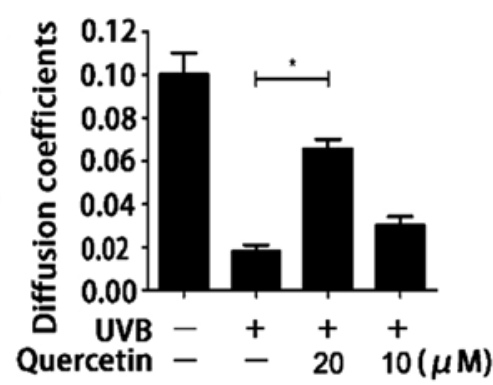

E

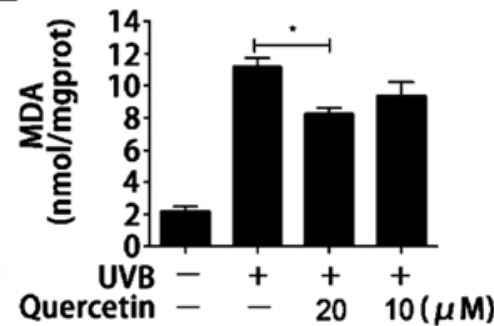

Figure 4. Effect of quercetin on cell membrane fluidity change and lipid peroxidation in the cultured HaCaT cells. HaCaT cells were pretreated with 10 or $20 \mu \mathrm{M}$ quercetin for $8 \mathrm{~h}$. Twenty-four hours post-irradiation, fluorescence recovery after photobleaching (FRAP) analysis was performed and the MDA levels were estimated. (A) Images before photobleaching, after photobleaching and after the recovery, respectively. The white circle indicates the photobleached region of the cell membrane. (B) The fluorescence intensity of the photobleached region in the differently treated cells. (a) No UVB treatment and no quercetin pretreatment; (b) UVB treatment and no quercetin pretreatment; (c) UVB treatment and $20 \mu \mathrm{M}$ quercetin pretreatment; (d) UVB treatment and $10 \mu \mathrm{m}$ quercetin pretreatment. (C) Recovery (R). (D) Effective diffusion coefficient (D). (E) Intracellular MDA levels. The data represent the means \pm SD of three independent experiments. ${ }^{*} \mathrm{P}<0.05,{ }^{* *} \mathrm{P}<0.01$.

are shown in Fig. 4A. The fluorescence intensity curves represent the membrane fluidity of the cells (Fig. 4B). UVB irradiation significantly reduced cell membrane fluidity, and pretreatment with quercetin at the concentration of $20 \mu \mathrm{M}$ markedly rescued cell membrane fluidity. The concentration of $20 \mu \mathrm{M}$ resulted in a more effective protective effect than the concentration of $10 \mu \mathrm{M}$. Fig. $4 \mathrm{C}$ and D show the recovery (R) and effective diffusion coefficient (D) of the cell membrane which suggested that quercetin had an obvious protective effect against ROS-induced cell membrane fluidity decrease.

Then, we further detected the lipid peroxidation of the cell membrane, which is the most common consequence of oxidative stress. MDA is a common indicator with which to determine the degree of lipid peroxidation. It is the final product of lipid peroxidation and is toxic to cells and the cell membrane $(10,32)$. Fig. 4E shows that UVB exposure 
A
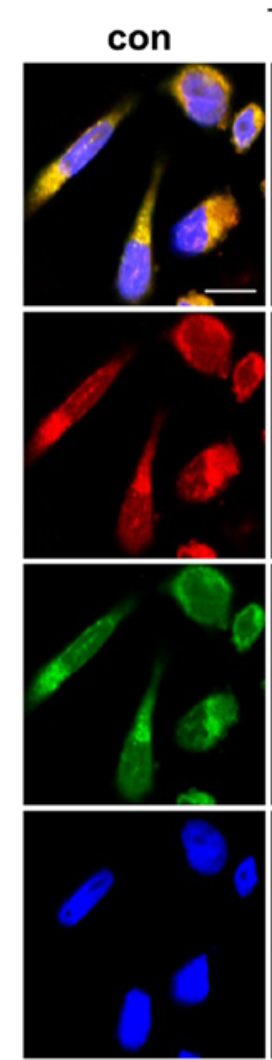

C
UVB $\left(15 \mathrm{~mJ} / \mathrm{cm}^{2}\right)$
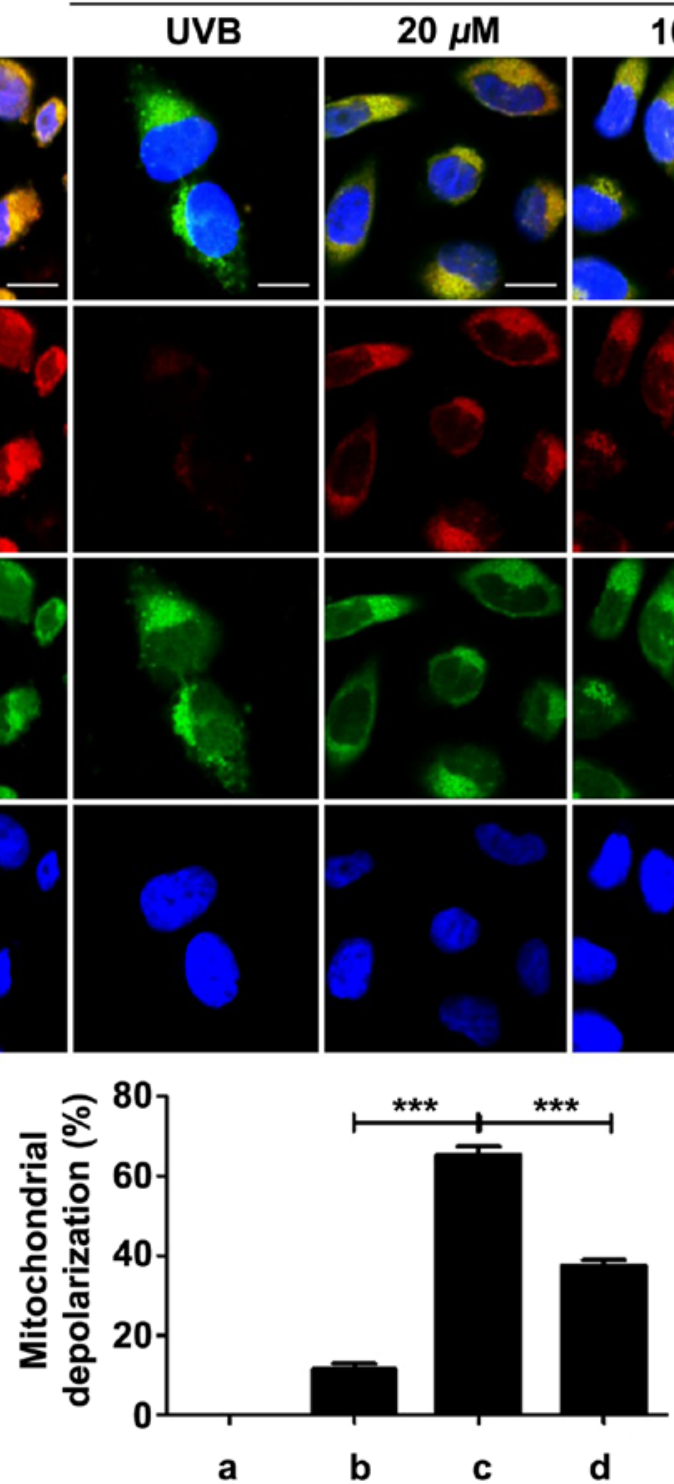

$\mathrm{Ba}$

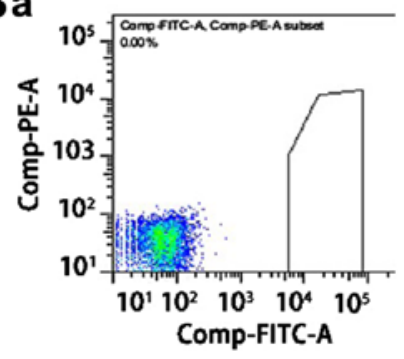

b



C

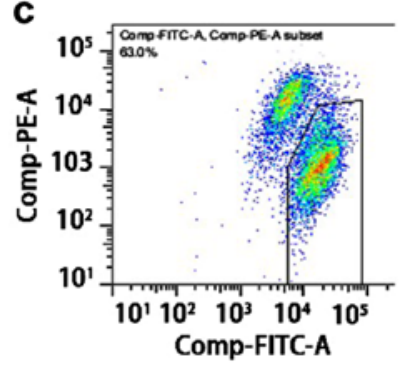

d

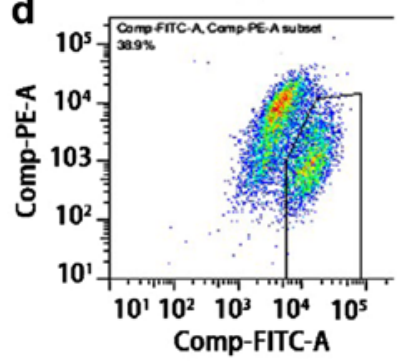

Figure 5. Effect of quercetin on mitochondrial membrane depolarization. HaCaT cells were pretreated with 10 or $20 \mu \mathrm{M}$ quercetin for 8 h. The mitochondrial membrane potential was monitored by staining with the fluorescent dye JC-1 at $24 \mathrm{~h}$ post-irradiation. Red fluorescence represents the mitochondrial aggregated form of JC-1 due to a high $\Delta \Psi \mathrm{m}$, whereas green fluorescence indicates the monomeric form of JC-1 due to low $\Delta \Psi \mathrm{m}$. (A) $\Delta \Psi \mathrm{m}$ was qualitatively determined via confocal laser scanning microscopy. Scale bars, $10 \mu \mathrm{m}$. (B) $\Delta \Psi \mathrm{m}$ was quantitatively determined via flow cytometry. (a) No JC-1 loaded; (b) JC-1 loaded and no UVB treatment; (c) JC-1 loaded and UVB treatment; (d) JC-1 loaded, UVB treatment and quercetin pretreatment. (C) The relative $\triangle \Psi \mathrm{m}$ is shown in the bar graph. The data represent the means \pm SD of three independent experiments. ${ }^{* * *} \mathrm{P}<0.001$.

induced an apparent increase in MDA and pretreatment with quercetin clearly inhibited the increase. The highest protective effect of quercetin was obtained at the concentration of $20 \mu \mathrm{M}$. The results suggest that quercetin effectively prevents cells from ROS-induced membrane fluidity decrease and lipid peroxidation.

Quercetin protects cells from mitochondrial damage. UVB irradiation induces a transient increase in the intracellular level of ROS, and the increased levels of ROS can attack the cell membrane and organelles, such as the mitochondria. Quercetin effectively prevented cells from ROS-induced cell membrane damage. Then, we investigated whether quercetin had a protective effect on preventing ROS-induced mitochondrial damage.
The integrity of $\Delta \Psi \mathrm{m}$ was assessed using JC-1 fluorescence staining and visualized via fluorescence microscopy. The red fluorescence decreased and the green fluorescence correspondingly increased after the UVB irradiation, indicating the disruption of $\Delta \Psi \mathrm{m}$ and mitochondrial dysfunction (Fig. 5A). Pretreatment with quercetin markedly rescued mitochondrial membrane depolarization. Fig. 5B shows the quantitative analysis of $\Delta \Psi \mathrm{m}$ and the bar graph in Fig. 5C displays the values of the flow cytometric results.

We further assessed the potential effect of ROS on mitochondrial morphology and the effect of quercetin on mitochondrial morphologic change. Tom 20 was used to identify mitochondrial morphology in each cell. Cells were classified into three groups. Cells classified as having tubular 
A
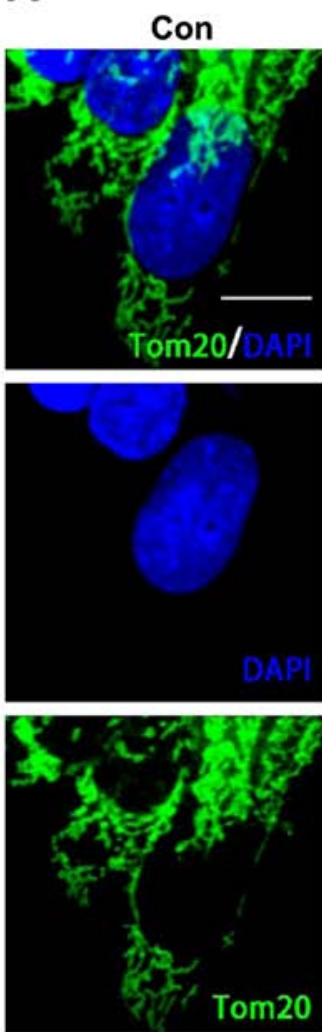

B
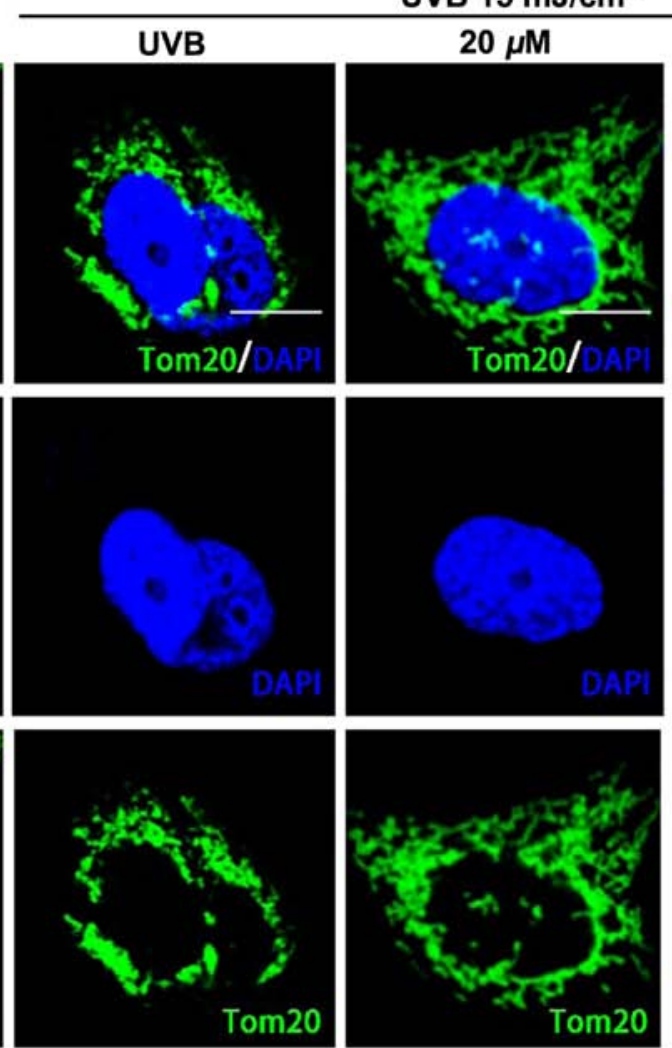

UVB $15 \mathrm{~mJ} / \mathrm{cm}^{2}$
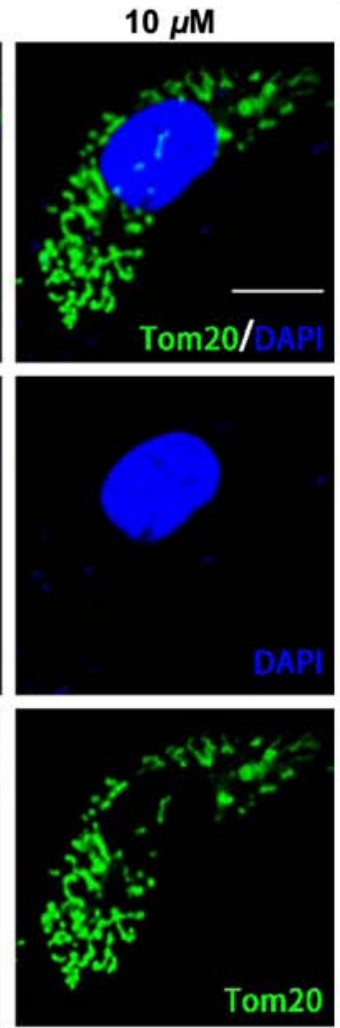

C
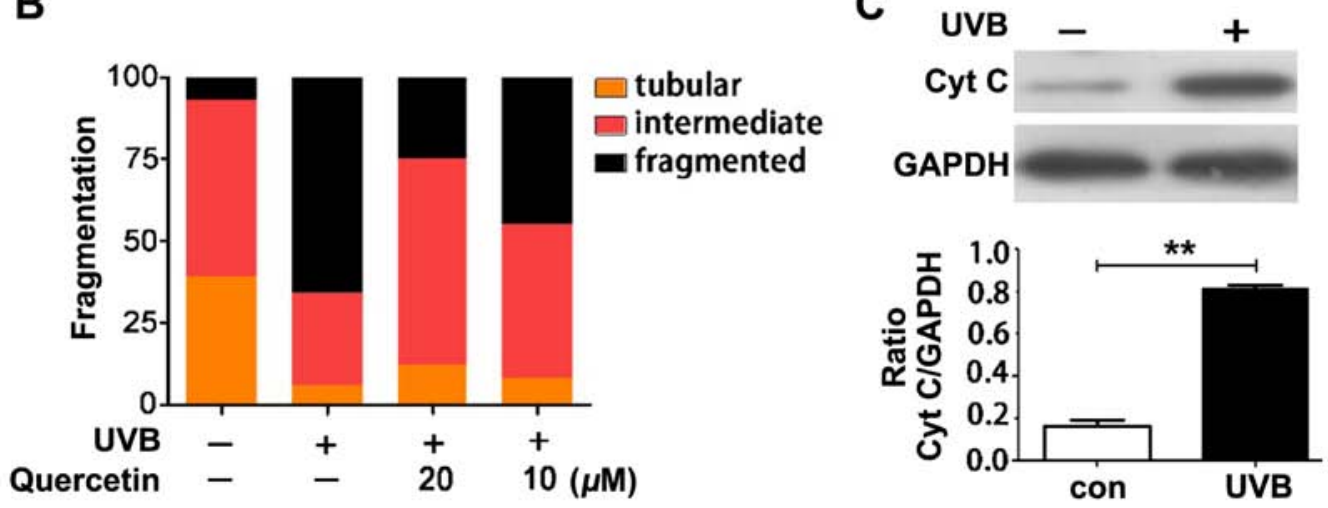

Figure 6. Effect of quercetin on mitochondrial morphological change. HaCaT cells were pretreated with 10 or $20 \mu \mathrm{M}$ quercetin for 8 h. Twenty-four hours post-irradiation, the mitochondrial morphology was observed by immunocytochemical staining. (A) Images of mitochondrial morphological change. Scale bars, $10 \mu \mathrm{m}$. (B) The percentage of cells with different mitochondrial morphology. Mitochondrial morphology was classified as fragmented, tubular, or intermediate. (C) The protein expression of cytochrome $c$ in mitochondrial damaged cells. The data represent the means \pm SD of three independent experiments. ${ }^{* *} \mathrm{P}<0.01$.

mitochondria contained almost entirely mitochondria with a length/width (axis) ratio $>10$. Cells classified as having fragmented mitochondria contained mitochondria with an axis ratio $<3$, and cells classified as having intermediate mitochondria contained both tubular and fragmented mitochondria $(10,33)$. Fig. 6A represents the morphology of the mitochondria in the differently treated cells. UVB irradiation interrupted tubular mitochondria and increased the numbers of fragmented mitochondria. Pretreatment with quercetin blocked ROS-induced mitochondrial fragmentation. Fig. 6B shows the numbers of cells with different mitochondrial morphology. The results suggest that quercetin protected cells from ROS-induced mitochondrial morphological change. Then, we further investigated whether the mitochondrial fragmentation accompanied the outflow of apoptosis-inducing factors, such as cytochrome $c$. Fig. 6C shows that the release of cytochrome $c$ occurred in the mitochondrial fragmented cells.

Quercetin blocks the release of cytochrome $c$ and inhibits apoptosis in the UVB-irradiated cells. UVB irradiation increases intracellular ROS levels and the increased ROS results in mitochondrial membrane depolarization, mitochondrial morphological change and the outflow of cytochrome $c$ from the mitochondria. The release of cytochrome $c$ into the cytosol can subsequently activates the caspase cascade that results in apoptosis. Thus, we examined the effect of quercetin on cytochrome $c$ release. Quercetin markedly inhibited the outflow of cytochrome $c$ from the mitochondria (Fig. 7A). Next, 
A

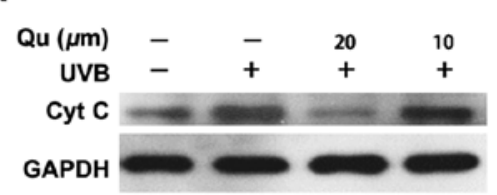

B

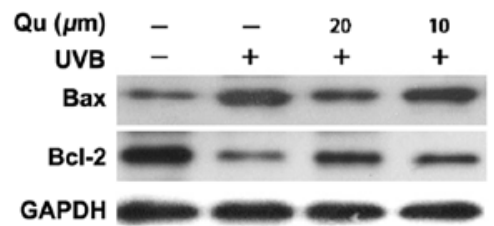

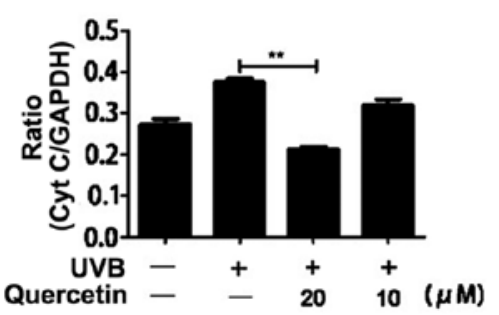

C
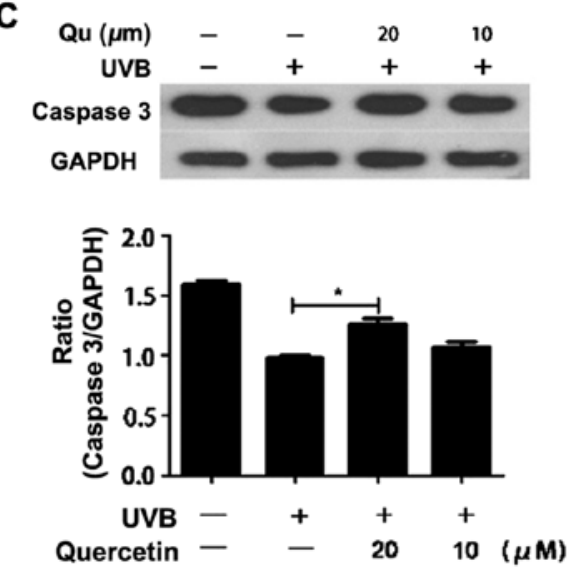

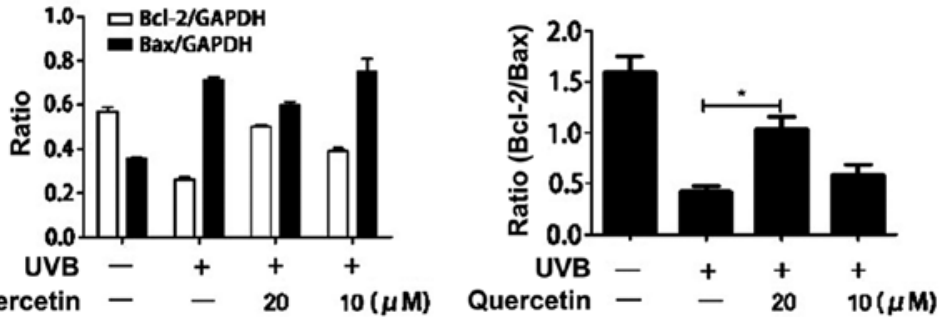

D

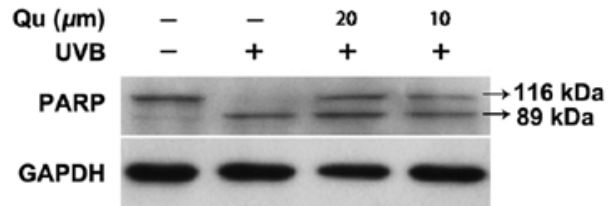

Figure 7. Quercetin blocks mitochondrial-dependent apoptosis. HaCaT cells were pretreated with 10 or $20 \mu \mathrm{M}$ quercetin for $8 \mathrm{~h}$. Twenty-four hours post-irradiation, protein expression was determined by western blot analysis: (A) cytochrome $c$, (B) Bcl-2 and Bax, (C) caspase-3 and (D) PARP. Quantitative analysis of proteins was conducted. The data represent the means $\pm \mathrm{SD}$ of three independent experiments. ${ }^{*} \mathrm{P}<0.05,{ }^{* *} \mathrm{P}<0.01$.

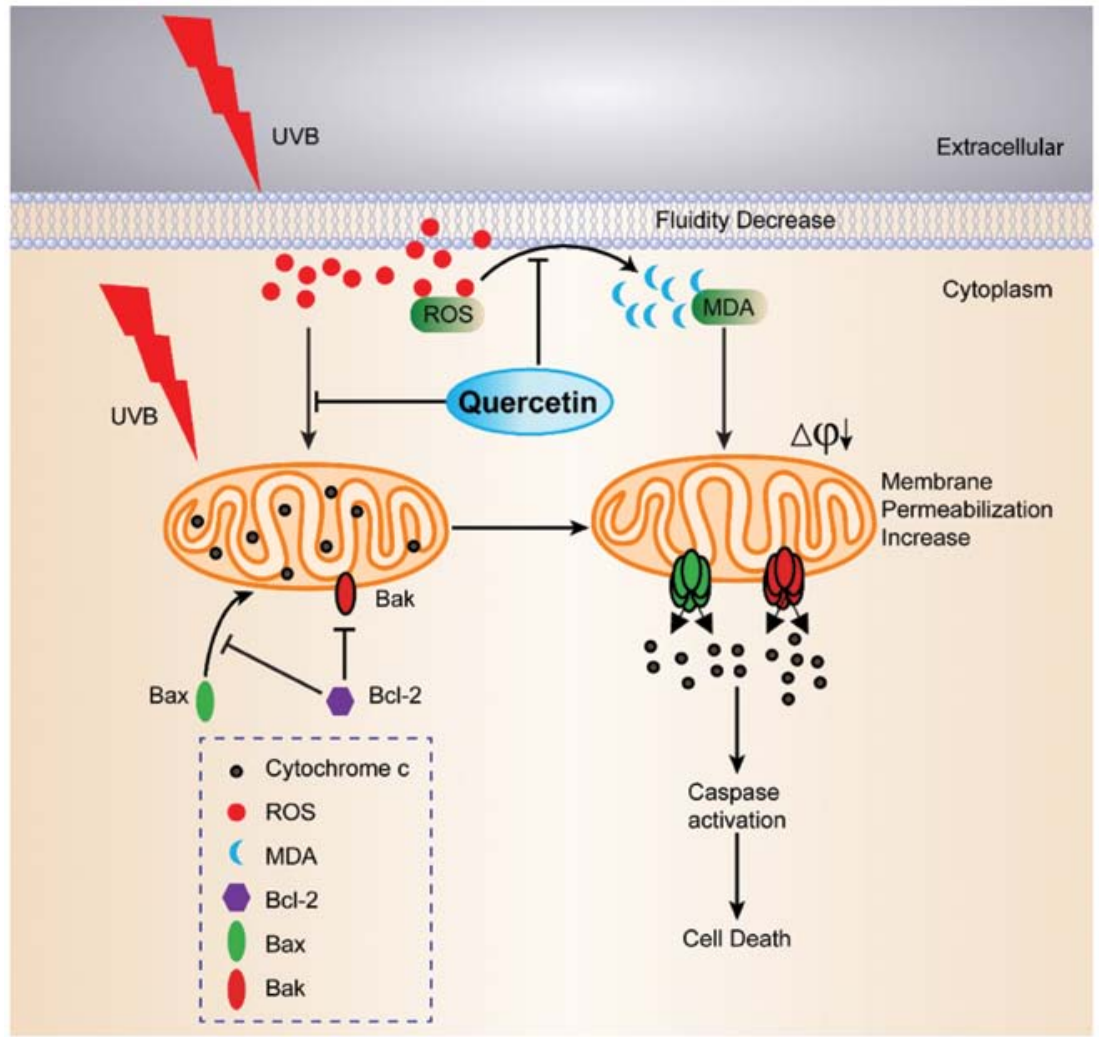

Figure 8. A pathway scheme displaying the proposed mechanism of the protective effect of quercetin against UVB irradiation-induced cell damage. 
we examined the effect of quercetin on the expression of Bcl-2 family proteins, which play an important role in the regulation of mitochondrial permeability, in the UVB-irradiated $\mathrm{HaCaT}$ cells. Western blot analysis revealed that pretreatment with quercetin increased the level of $\mathrm{Bcl}-2$ and decreased the level of Bax in the UVB-irradiated HaCaT cells. Therefore, the ratio of Bax to Bcl-2 was increased (Fig. 7B). The caspase family is believed to play a central role in mediating various apoptotic responses. To confirm the induction of the apoptotic pathway, we examined the activation of caspase-3 and PARP. Pretreatment with quercetin induced a significant increase in caspase-3 after UVB irradiation (Fig. 7C and D). PARP, which is a substrate of caspase-3, was cleaved in the quercetin-pretreated cells in response to UVB irradiation. These findings suggest that quercetin protects cells against UVB irradiation-induced cell death via the mitochondrial-mediated apoptotic pathway.

In conclusion, the present study demonstrated that quercetin can effectively scavenge intracellular ROS, and preincubation with quercetin blocked ROS attack on the cell membrane and mitochondria after UVB exposure. The decrease in cell membrane fluidity was inhibited by quercetin. The integrity of $\Delta \Psi \mathrm{m}$ was rescued and the mitochondrial morphology was protected. Moreover, the release of cytochrome $c$ was prevented and an elevation in the $\mathrm{Bcl}-2 / \mathrm{Bax}$ ratio was observed. These data indicate that quercetin prevented the cell membrane and mitochondria from ROS-induced damage and blocked the mitochondrial-dependent apoptotic pathway. The ROS clearing ability of quercetin provides protection against UVB irradiation-induced apoptosis of HaCaT cells (Fig. 8). In addition, ROS clearing agent NAC effectively inhibited UVB-induced apoptosis (Fig. 3C) and further proved that the protective effect of quercetin on UVB-induced cell death was associated with ROS clearing ability. These findings suggest that quercetin exerts beneficial effects against UVB irradiation-induced skin photo-damage.

\section{Acknowledgements}

The present study was supported by a grant to XJ.S. from the 'Shenzhen Hong Kong Innovation Circle' Research Program (ZYB200907100147A) and the Novel Innovation Project of Graduate School at Shenzhen, Tsinghua University (JZ2016000).

\section{References}

1. Paz ML, González Maglio DH, Weill FS, Bustamante J and Leoni J: Mitochondrial dysfunction and cellular stress progression after ultraviolet $\mathrm{B}$ irradiation in human keratinocytes. Photodermatol Photoimmunol Photomed 24: 115-122, 2008.

2. Katiyar SK, Matsui MS, Elmets CA and Mukhtar H: Polyphenolic antioxidant (-)-epigallocatechin-3-gallate from green tea reduces UVB-induced inflammatory responses and infiltration of leukocytes in human skin. Photochem Photobiol 69: 148-153, 1999.

3. Hanson KM and Clegg RM: Observation and quantification of ultraviolet-induced reactive oxygen species in ex vivo human skin. Photochem Photobiol 76: 57-63, 2002.

4. Beak SM, Lee YS and Kim JA: NADPH oxidase and cyclooxygenase mediate the ultraviolet $\mathrm{B}$-induced generation of reactive oxygen species and activation of nuclear factor-kappaB in $\mathrm{HaCaT}$ human keratinocytes. Biochimie 86: 425-429, 2004.
5. Sander CS, Chang H, Hamm F, Elsner P and Thiele JJ: Role of oxidative stress and the antioxidant network in cutaneous carcinogenesis. Int J Dermatol 43: 326-335, 2004.

6. Bickers DR and Athar M: Oxidative stress in the pathogenesis of skin disease. J Invest Dermatol 126: 2565-2575, 2006.

7. Black HS: Potential involvement of free radical reactions in ultraviolet light-mediated cutaneous damage. Photochem Photobiol 46: 213-221, 1987.

8. Wlaschek M, Tantcheva-Poór I, Naderi L, Ma W, Schneider LA, Razi-Wolf Z, Schüller J and Scharffetter-Kochanek K: Solar UV irradiation and dermal photoaging. J Photochem Photobiol B 63: 41-51, 2001.

9. Heck DE, Vetrano AM, Mariano TM and Laskin JD: UVB light stimulates production of reactive oxygen species: Unexpected role for catalase. J Biol Chem 278: 22432-22436, 2003.

10. Abdul-Muneer PM, Chandra N and Haorah J: Interactions of oxidative stress and neurovascular inflammation in the pathogenesis of traumatic brain injury. Mol Neurobiol 51: 966-979, 2014.

11. Tavolari S, Munarini A, Storci G, Laufer S, Chieco P and Guarnieri T: The decrease of cell membrane fluidity by the non-steroidal anti-inflammatory drug Licofelone inhibits epidermal growth factor receptor signalling and triggers apoptosis in HCA-7 colon cancer cells. Cancer Lett 321: 187-194, 2012.

12. Orrenius S, Gogvadze V and Zhivotovsky B: Mitochondrial oxidative stress: Implications for cell death. Annu Rev Pharmacol Toxicol 47: 143-183, 2007.

13. Segre JA: Epidermal barrier formation and recovery in skin disorders. J Clin Invest 116: 1150-1158, 2006.

14. Morrow DMP, Fitzsimmons PEE, Chopra M and McGlynn H: Dietary supplementation with the anti-tumour promoter quercetin: Its effects on matrix metalloproteinase gene regulation. Mutat Res 480-481: 269-276, 2001.

15. Galati G, Moridani MY, Chan TS and O'Brien PJ: Peroxidative metabolism of apigenin and naringenin versus luteolin and quercetin: Glutathione oxidation and conjugation. Free Radic Biol Med 30: 370-382, 2001.

16. Sekher Pannala A, Chan TS, O'Brien PJ and Rice-Evans CA: Flavonoid B-ring chemistry and antioxidant activity: Fast reaction kinetics. Biochem Biophys Res Commun 282: 1161-1168, 2001.

17. Sawa T, Nakao M, Akaike T, Ono K and Maeda H: Alkylperoxyl radical-scavenging activity of various flavonoids and other phenolic compounds: Implications for the anti-tumor-promoter effect of vegetables. J Agric Food Chem 47: 397-402, 1999.

18. Ioku K, Tsushida T, Takei Y, Nakatani N and Terao J: Antioxidative activity of quercetin and quercetin monoglucosides in solution and phospholipid bilayers. Biochim Biophys Acta 1234: 99-104, 1995.

19. Bonina F, Lanza M, Montenegro L, Puglisi C, Tomaino A, Trombetta D, Castelli F and Saija A: Flavonoids as potential protective agents against photo-oxidative skin damage. Int J Pharm 145: 87-94, 1996.

20. Morel I, Lescoat G, Cogrel P, Sergent O, Pasdeloup N, Brissot P, Cillard $\mathrm{P}$ and Cillard J: Antioxidant and iron-chelating activities of the flavonoids catechin, quercetin and diosmetin on iron-loaded rat hepatocyte cultures. Biochem Pharmacol 45: 13-19, 1993.

21. Casagrande R, Georgetti SR, Verri WA Jr,Dorta DJ, dos Santos AC and Fonseca MJV: Protective effect of topical formulations containing quercetin against UVB-induced oxidative stress in hairless mice. J Photochem Photobiol B 84: 21-27, 2006.

22. Casagrande R, Georgetti SR, Verri WA Jr, Jabor JR, Santos AC and Fonseca MJV: Evaluation of functional stability of quercetin as a raw material and in different topical formulations by its antilipoperoxidative activity. AAPS PharmSciTech 7: E10, 2006.

23. Goodwin JS and Kenworthy AK: Photobleaching approaches to investigate diffusional mobility and trafficking of Ras in living cells. Methods 37: 154-164, 2005.

24. Pucadyil TJ and Chattopadhyay A: Effect of cholesterol on lateral diffusion of fluorescent lipid probes in native hippocampal membranes. Chem Phys Lipids 143: 11-21, 2006.

25. Axelrod D, Koppel DE, Schlessinger J, Elson E and Webb WW: Mobility measurement by analysis of fluorescence photobleaching recovery kinetics. Biophys J 16: 1055-1069, 1976.

26. Høyer-Hansen M, Bastholm L, Szyniarowski P, Campanella M, Szabadkai G, Farkas T, Bianchi K, Fehrenbacher N, Elling F, Rizzuto R, et al: Control of macroautophagy by calcium, calmodulin-dependent kinase kinase-beta, and Bcl-2. Mol Cell 25: 193-205, 2007. 
27. Rice-Evans CA, Miller NJ and Paganga G: Structure-antioxidant activity relationships of flavonoids and phenolic acids. Free Radic Biol Med 20: 933-956, 1996.

28. Skaper SD, Fabris M, Ferrari V, Dalle Carbonare M and Leon A: Quercetin protects cutaneous tissue-associated cell types including sensory neurons from oxidative stress induced by glutathione depletion: Cooperative effects of ascorbic acid. Free Radic Biol Med 22: 669-678, 1997.

29. Svobodova A, Walterova D and Vostalova J: Ultraviolet light induced alteration to the skin. Biomed Pap Med Fac Univ Palacky Olomouc Czech Repub 150: 25-38, 2006.

30. Shindo Y and Hashimoto T: Ultraviolet B-induced cell death in four cutaneous cell lines exhibiting different enzymatic antioxidant defences: Involvement of apoptosis. J Dermatol Sci 17: 140-150, 1998
31. LeBel CP, Ischiropoulos $\mathrm{H}$ and Bondy SC: Evaluation of the probe 2',7'-dichlorofluorescin as an indicator of reactive oxygen species formation and oxidative stress. Chem Res Toxicol 5: 227-231, 1992.

32. Millán-Plano S, García JJ, Martínez-Ballarín E, Reiter RJ, Ortega-Gutiérrez S, Lázaro RM and Escanero JF: Melatonin and pinoline prevent aluminium-induced lipid peroxidation in rat synaptosomes. J Trace Elem Med Biol 17: 39-44, 2003.

33. Nakamura K, Nemani VM, Azarbal F, Skibinski G, Levy JM, Egami K, Munishkina L,Zhang J, Gardner B, Wakabayashi J, et al: Direct membrane association drives mitochondrial fission by the Parkinson disease-associated protein alpha-synuclein. J Biol Chem 286: 20710-20726, 2011. 